\title{
Spaces with maximal projection constants
}

\author{
by \\ HERMANN KÖNIG (Kiel) and \\ Nicole TOMCZAK-JAEGERMANN (Edmonton) \\ Dedicated to Olek Pelczyński on the occasion of his 70th birthday \\ with thanks for all his questions
}

\begin{abstract}
We show that $n$-dimensional spaces with maximal projection constants exist not only as subspaces of $l_{\infty}$ but also as subspaces of $l_{1}$. They are characterized by a rigid set of vector conditions. Nevertheless, we show that, in general, there are many non-isometric spaces with maximal projection constants. Several examples are discussed in detail.
\end{abstract}

1. Spaces with maximal projection constants. In this paper we study the question of non-uniqueness of finite-dimensional spaces with maximal projection constant and their imbeddings into $l_{\infty}$ and $l_{1}$. Given a (closed) subspace $X$ of a Banach space $Z$, the relative projection constant of $X$ in $Z$ is

$$
\lambda(X, Z):=\inf \{\|P\| \mid P: Z \rightarrow X \text { is a linear projection onto } X\},
$$

and the (absolute) projection constant of $X$ is

$$
\lambda(X):=\sup \{\lambda(X, Z) \mid Z \text { a Banach space containing } X \text { as a subspace }\} .
$$

The scalar field $\mathbb{K}$ will be either the reals $\mathbb{R}$ or the complex numbers $\mathbb{C}$. Any separable Banach space can be imbedded into $l_{\infty}$; for any such imbed$\operatorname{ding} \lambda(X)=\lambda\left(X, l_{\infty}\right), l_{\infty}$ is the natural superspace. For finite-dimensional spaces, $\lambda(X) \leq \sqrt{\operatorname{dim} X}$ by Kadets-Snobar [KS].

In fact, more is known: Let $1<n<N<\infty$ and

$$
\begin{aligned}
f_{\mathbb{K}}(n, N) & :=\sup \{\lambda(X, Z) \mid X \subseteq Z, \operatorname{dim} X=n, \operatorname{dim} Z=N\}, \\
g_{\mathbb{K}}(n) & :=\sup \{\lambda(X) \mid \operatorname{dim} X=n\} .
\end{aligned}
$$

2000 Mathematics Subject Classification: Primary 46B20.

N. Tomczak-Jaegermann holds the Canada Research Chair in Geometric Analysis. 
Further, let us introduce

$$
\begin{aligned}
F(n, N) & :=\sqrt{n}([\sqrt{n}+\sqrt{(N-1)(N-n)}] / N), \\
G_{\mathbb{R}}(n) & :=[2+(n-1) \sqrt{n+2}] /(n+1), \\
G_{\mathbb{C}}(n) & :=[1+(n-1) \sqrt{n+1}] /(n) .
\end{aligned}
$$

Then by [KLL] and [KT2] for all $n<N$ one has

$$
\begin{aligned}
f_{\mathbb{K}}(n, N) & \leq F(n, N), \\
g_{\mathbb{K}}(n) & \leq G_{\mathbb{K}}(n) .
\end{aligned}
$$

We note that $F(n, N), G_{\mathbb{K}}(n)<\sqrt{n}$ and, in fact,

$$
G_{\mathbb{R}}(n)=F(n, n(n+1) / 2), \quad G_{\mathbb{C}}(n)=F\left(n, n^{2}\right) .
$$

An $n$-dimensional subspace $X_{n} \subseteq l_{\infty}^{N}$ can be given by a basis $\left(f_{j}\right)_{j=1}^{n}$ where $f_{j}=\left(f_{j s}\right)_{s=1}^{N} \in \mathbb{K}^{N}$. Writing the coordinates of $\widetilde{x}=\sum_{j=1}^{n} x_{j} f_{j} \in X_{n}$ as $x=\left(x_{j}\right)_{j=1}^{n}$, we have

$$
\|\widetilde{x}\|=\left\|\sum_{j=1}^{n} x_{j} f_{j}\right\|_{\infty}=\sup _{1 \leq s \leq N}\left|\left\langle x, x_{s}\right\rangle\right|=:\|x\|
$$

where $x_{s}=\left(f_{j s}\right)_{j=1}^{n} \in \mathbb{K}^{n}$ and $\langle\cdot, \cdot\rangle$ is the usual scalar product in $\mathbb{K}^{n}$. We identify in the following $\left(\mathbb{K}^{n},\|\cdot\| \|\right)$ with $X_{n} \subseteq l_{\infty}^{N}$ and write $\|x\|$ instead of $\|\widetilde{x}\|$, both spaces being isometric.

A very rigid set of conditions needs to be imposed on the vectors $x_{s} \in \mathbb{K}^{n}$ $(s=1, \ldots, N)$ to have equality in $(1.1)$ or $(1.2)$, i.e. $\lambda\left(X_{n}, l_{\infty}^{N}\right)=F(n, N)$ or $\lambda(X)=G_{\mathbb{K}}(n)$ : the vectors have to form a tight spherical 4-design (see [KT2]). In spite of this being a very strong assumption, we will show that - in general - there are many non-isometric spaces with maximal projection constant, some of them even being realized as subspaces of $l_{1}$. In a recent paper, Chalmers and Lewicki $[\mathrm{CL}]$ show that among the symmetric sequence spaces with maximal projection constants there are symmetric subspaces of $l_{1}$. This result motivated a part of the current paper.

We use standard Banach space terminology (see e.g. [TJ]). In particular, $l_{p}^{N}$ denotes $\mathbb{K}^{N}$ with the $p$-norm if $N \in \mathbb{N}$ and $1 \leq p \leq \infty$. Given a measure $\mu$ on $\{1, \ldots, N\}, l_{p}^{N}(\mu)$ denotes $\mathbb{K}^{N}$, equipped with the norm $\|x\|=\left(\sum_{s=1}^{N}\left|x_{s}\right|^{p} \mu_{s}\right)^{1 / p}, x=\left(x_{s}\right)_{s=1}^{N} \in \mathbb{K}^{N}$. The Banach-Mazur distance between two $n$-dimensional normed spaces $X$ and $Y$ is given by

$$
d(X, Y)=\inf \left\{\|T\|\left\|T^{-1}\right\| \mid T: X \rightarrow Y \text { is a linear isomorphism }\right\} .
$$

Our main results are:

Theorem 1. Let $n \in \mathbb{N}, N \in \mathbb{N} \cup\{\infty\}$ and $X_{n} \subseteq l_{\infty}^{N}$ be a space such that $X_{n}$ has maximal projection constant among all $n$-dimensional spaces. 
Thus $\lambda\left(X_{n}\right)=g_{\mathbb{K}}(n)$. Then there is $Y_{n} \subseteq l_{1}^{N}$ also having maximal projection constant

$$
\lambda\left(Y_{n}\right)=\lambda\left(Y_{n}, l_{1}^{N}\right)=\lambda\left(X_{n}\right)=g_{\mathbb{K}}(n) .
$$

$A$ corresponding fact holds for relative projection constants: if $X_{n} \subset l_{\infty}^{N}$ satisfies $\lambda\left(X_{n}, l_{\infty}^{N}\right)=f_{\mathbb{K}}(n, N)$ for $N \in \mathbb{N}$, there is $Y_{n} \subseteq l_{1}^{N}$ with

$$
\lambda\left(Y_{n}, l_{1}^{N}\right)=\lambda\left(X_{n}, l_{\infty}^{N}\right)=f_{\mathbb{K}}(n, N) .
$$

In the real two-dimensional case, $\lambda\left(X_{2}\right)=g(2)=f(2,3)=4 / 3$ is uniquely attained by the space $X_{2}$ having the regular hexagon as its unit ball, and $Y_{2}=X_{2}$ holds isometrically. Both spaces are represented in $\mathbb{R}^{3}$ with the $\|\cdot\|_{\infty^{-}}$or $\|\cdot\|_{1^{-n o r m}}$ by the hyperplane $H=\left\{x=\left(x_{j}\right)_{j=1}^{3} \in \mathbb{R}^{3} \mid\right.$ $\left.\sum_{j=1}^{3} x_{j}=0\right\}$. In general, however, spaces $X_{n} \subseteq l_{\infty}^{N}$ and $Y_{n} \subseteq l_{1}^{N}$ with maximal projection constant are not isometric. This already occurs for $n=3$ in the real and $n=2$ in the complex case.

Proposition 2. Let $D$ be the dodecahedron in $\mathbb{R}^{3}$ and $I$ be the icosahedron having as its vertices the midpoints of the faces of $D$. Let $K=D \cap \phi I$ where $\phi=(1+\sqrt{5}) / 2$. Let $X_{3}$ and $Y_{3}$, respectively, be the 3 -dimensional spaces having $D$ and $K$ as their unit balls. Then $X_{3} \subseteq l_{\infty}^{6}, Y_{3} \subseteq l_{1}^{6}$ and both have maximal projection constant

$$
\lambda\left(X_{3}\right)=\lambda\left(Y_{3}\right)=\lambda\left(Y_{3}, l_{1}^{6}\right)=G_{\mathbb{R}}(3)=F(3,6)=\phi .
$$

$A$ similar example exists for $\mathbb{K}=\mathbb{C}$ and $n=2$.

Clearly $Y_{3}$ and $X_{3}$ are non-isometric, $Y_{3}$ having 12 regular pentagons and 20 regular triangles as its faces. There are infinitely many non-isometric spaces with unit balls between $K$ and $D$ having maximal projection constant $\phi$.

Known examples of $n$-dimensional spaces with maximal projection constant are often realized as subspaces of $l_{\infty}^{N}$ where $N \sim n^{2}$. In this kind of situation we can always find many non-isometric spaces with extremal projection constant, $n$ being sufficiently large.

TheOREM 3. Let $n>2$ and $8 \leq N \leq e^{\sqrt{n} /(8 e)}$. If $X_{n} \subseteq l_{\infty}^{N}$ is an $n$ dimensional space with maximal projection constant, $\lambda\left(X_{n}\right)=g_{\mathbb{K}}(n)$, there are infinitely many mutually non-isometric $n$-dimensional spaces $Y_{n}$ with

$$
\lambda\left(X_{n}\right)=\lambda\left(Y_{n}\right)=g_{\mathbb{K}}(n) .
$$

These spaces are constructed by probabilistic methods. There is, however, an asymptotic sequence of spaces defined more explicitly exhibiting a similar property to the one in Proposition 2.

Proposition 4. Let $n=p^{m}$ be an odd prime power and set $N=$ $n^{2}-n+1$. Then there exist complex $n$-dimensional subspaces of $\mathbb{C}^{N}$, which 
we call $X_{n}$ and $Y_{n}$ when considered as subspaces of $l_{\infty}^{N}$ and $l_{1}^{N}$, respectively, satisfying:

(a) $X_{n}$ and $Y_{n}$ have extremal relative projection constant $\lambda\left(X_{n}, l_{\infty}^{N}\right)=$ $\lambda\left(Y_{n}, l_{1}^{N}\right)=f_{\mathbb{C}}(n, N)=F(n, N)$,

(b) $X_{n}$ and $Y_{n}$ are non-isometric. In fact, the Banach-Mazur distance to $l_{2}^{n}$ satisfies $d\left(X_{n}, l_{2}^{n}\right)=\sqrt{n}, d\left(Y_{n}, l_{2}^{n}\right) \leq \sqrt{2}$.

We note that the absolute projection constants of $X_{n}$ are almost the maximal possible ones since $\lambda\left(X_{n}\right) \leq G(n)$ always holds and $G(n)-F(n, N) \leq$ $1 /\left(2 n^{3 / 2}\right), F(n, N) \geq \sqrt{n}-1 /(2 \sqrt{n})$.

2. Characterization of extremal cases. The proofs of our theorems rely on the following duality result:

Proposition 5. Let $N \in \mathbb{N} \cup\{\infty\}$ and $n \in \mathbb{N}, n<N$. Then

$$
\begin{aligned}
\sup \left\{\lambda\left(Z_{n}, l_{\infty}^{N}\right) \mid Z_{n} \text { is an } n \text {-dimensional subspace of } l_{\infty}^{N}\right\} & \\
& =n \sup \left\{\sum_{s, t=1}^{N} \mu_{s} \mu_{t}||\left\langle x_{s}, x_{t}\right\rangle \mid\right\}=: \Lambda
\end{aligned}
$$

where the second supremum is taken over all discrete probability measures $\mu=\left(\mu_{s}\right)_{s=1}^{N}$ on $\{1, \ldots, N\}$ or $\mathbb{N},\|\mu\|_{1}=1$, and over all sets of vectors $x_{s} \in S^{n-1}(\mathbb{K}), s=1, \ldots, N$, such that

$$
\operatorname{Id}_{n}=n \sum_{t=1}^{N} \mu_{t}\left\langle\cdot, x_{t}\right\rangle x_{t} \quad\left(\text { on } \mathbb{K}^{n}\right) .
$$

Both suprema are, in fact, maxima. Given extremal elements $\left(x_{t}, \mu_{t}\right)$ attaining $\Lambda$, let $S:=\operatorname{supp} \mu$ and $M:=|S| \leq N$. Then an $n$-dimensional space $X_{n} \subseteq l_{\infty}^{M}$ with maximal projection constant $\Lambda$ is given by its norm

$$
\|x\|:=\sup _{s \in S}\left|\left\langle x, x_{s}\right\rangle\right|, \quad x \in \mathbb{K}^{n} .
$$

The dual unit ball of $X_{n}$ is the absolutely convex hull of the vectors $\left(x_{s}\right)_{s \in S}$. Further, $\Lambda=\sum_{t=1}^{N} \mu_{t}\left|\left\langle x_{s}, x_{t}\right\rangle\right|$ is independent of $s \in S$, and the formula $u=\left(\operatorname{sgn}\left(\left\langle x_{s}, x_{t}\right\rangle\right) \mu_{t}\right)_{s, t \in S}$ defines a map on $l_{\infty}^{M}$ with $\left.u\right|_{X_{n}}=(\Lambda / n) \operatorname{Id}_{X_{n}}$.

Proposition 5 is essentially a consequence of proofs in [KT2] except for some lemma which was formulated there under an additional but unnecessary condition. To formulate the improved version, for $n \in \mathbb{N}$ and $N \in \mathbb{N} \cup\{\infty\}$ set $T=\{1, \ldots, N\}$ and

$$
\varphi(n, T)=\sup \sum_{s, t \in T}\left|\sum_{j=1}^{n} f_{j}(s) \overline{f_{j}(t)}\right| \mu_{s} \mu_{t}
$$


where the supremum is extended over all probability measures $\mu=\left(\mu_{s}\right)_{s=1}^{N}$ on $T$ and all orthonormal systems $\left(f_{j}\right)_{j=1}^{n}$ of length $n$ in $l_{2}^{N}(\mu)$.

Lemma 6. Assume that $\mu^{\circ}=\left(\mu_{s}^{\circ}\right)_{s=1}^{N}$ and $f_{1}^{\circ}, \ldots, f_{n}^{\circ}$ attain the supremum

$$
\varphi(n, T)=\sum_{s, t \in T}\left|\sum_{j=1}^{n} f_{j}^{\circ}(s) \overline{f_{j}^{\circ}(t)}\right| \mu_{s}^{\circ} \mu_{t}^{\circ} .
$$

Then for all $l, m=1, \ldots, n$, there exists a sequence $1 \leq l_{0}, \ldots, l_{k} \leq n$ such that $l_{0}=l, l_{k}=m$ and for any $1 \leq r \leq k$ we have

$$
\operatorname{supp} f_{l_{r-1}}^{\circ} \cap \operatorname{supp} f_{l_{r}}^{\circ} \cap \operatorname{supp} \mu^{\circ} \neq \emptyset \text {. }
$$

Proof. For $0<\tau \leq 1$, let $\mathcal{M}_{\tau}$ denote the set of all discrete measures on $T$ such that $\mu(T)=\tau$. By $\varphi(n, T, \tau)$ we denote the supremum analogous to $\varphi(n, T)$ except that $\mu \in \mathcal{M}_{\tau}$, the orthonormalization of the $f_{j}$ 's being taken with respect to $\mu / \tau$. Then $\varphi(n, T)=\varphi(n, T, 1)$ and $\varphi(n, T, \tau)=\tau^{2} \varphi(n, T, 1)$. Further $\varphi\left(n_{1}, T_{1}, 1\right) \leq \varphi(n, T, 1)$ if $n_{1} \leq n$ and $T_{1} \subseteq T$. Assume that $\mu^{\circ}$ and $f_{1}^{\circ}, \ldots, f_{n}^{\circ}$ attain the supremum $\varphi(n, T)$. Let $J_{1} \subseteq\{1, \ldots, n\}$ be a maximal set with the following property: $J_{1}=\left\{j_{1}, \ldots, j_{\varrho}\right\}$ and for every $1<r \leq \varrho$ we have

$$
\operatorname{supp} f_{j_{r}}^{\circ} \cap \bigcup_{l=1}^{r-1} \operatorname{supp} f_{j_{l}}^{\circ} \cap \operatorname{supp} \mu^{\circ} \neq \emptyset .
$$

Let $J_{2}=\{1, \ldots, n\} \backslash J_{1}$. Moreover, put $T_{1}=\bigcup_{j \in J_{1}} \operatorname{supp} f_{j}^{\circ} \cap \operatorname{supp} \mu^{\circ}$ and $T_{2}=T-T_{1}$. Then

$$
f_{j}^{\circ}(s) \mu_{s}=0 \quad \text { if }(s, j) \in\left(T_{2} \times J_{1}\right) \cup\left(T_{1} \times J_{2}\right) .
$$

Indeed, for $j \in J_{1}$ and $s \in T_{2}$ this follows from the definition of $T_{2}$. For $j \in J_{2}$ and $s \in T_{1}$ this is a consequence of the maximality of $J_{1}$ since otherwise $J_{1} \cup\{j\}$ would satisfy (2.3).

The definition of $J_{1}$ and an easy induction show that $m \in J_{1}$ if and only if there exists a finite sequence joining $j_{1}$ and $m$, i.e. a sequence $l_{0}, \ldots, l_{k}$ in $J_{1}$ with $l_{0}=j_{1}$ and $l_{k}=m$ such that

$$
\operatorname{supp} f_{l_{r}} \cap \operatorname{supp} f_{l_{r-1}} \cap \operatorname{supp} \mu^{\circ} \neq \emptyset \quad \text { for all } 1 \leq r \leq k .
$$

If $l, m \in J_{1}$ are arbitrary, a similar sequence satisfying the conclusion of the lemma is obtained by concatenating sequences joining $l$ with $j_{1}$ and $j_{1}$ with $m$.

Finally, we show that the maximality assumption defining $J_{1}$ implies that $J_{1}=\{1, \ldots, n\}$. Let $n_{i}:=\left|J_{i}\right|$ and $\tau_{i}:=\sum_{s \in T_{i}} \mu_{s}^{\circ}$ for $i=1,2$. Thus $n=n_{1}+n_{2}$ and $\tau_{1}+\tau_{2}=1$. For $I \subseteq J:=\{1, \ldots, n\}$ and $U \subseteq T$ define

$$
\phi(I, U)=\sum_{s, t \in U}\left|\sum_{i \in I} f_{i}^{\circ}(s) \overline{f_{i}^{\circ}(t)}\right| \mu_{s}^{\circ} \mu_{t}^{\circ} .
$$


Then, by (2.4),

$$
\begin{aligned}
\varphi(n, T) & =\phi(J, T)=\phi\left(J_{1}, T_{1}\right)+\phi\left(J_{2}, T_{2}\right) \\
& \leq \varphi\left(n_{1}, T_{1}, \tau_{1}\right)+\varphi\left(n_{2}, T_{2}, \tau_{2}\right)=\tau_{1}^{2} \varphi\left(n_{1}, T_{1}\right)+\tau_{2}^{2} \varphi\left(n_{2}, T_{2}\right) \\
& \leq\left(\tau_{1}^{2}+\tau_{2}^{2}\right) \varphi(n, T) .
\end{aligned}
$$

Thus $\tau_{1}^{2}+\tau_{2}^{2} \geq 1$ and hence $\tau_{1}=1, \tau_{2}=0$ since $\tau_{1}+\tau_{2}=1$, and $\tau_{1}>0$ since $J_{1} \neq \emptyset$ and $T_{1} \neq \emptyset$. This implies that $T_{1}=\operatorname{supp} \mu^{\circ}$ and, by the maximality of $J_{1}$, that $J_{1}=J$.

We will need the nuclear norm $\nu$ on spaces of finite rank operators between Banach spaces and the fact that the trace of a finite rank operator $T \in \mathcal{L}(X)$ can be estimated by $|\operatorname{tr}(s)| \leq \nu(s)$; cf. e.g. [TJ].

Proof of Proposition 5. We indicate how the statements in Proposition 5 follow from the results and proofs in [KT2] and Lemma 6.

Let $T=\{1, \ldots, N\}$ if $N \in \mathbb{N}$ or $T=\mathbb{N}$ if $N=\infty$. Let $X_{n} \subseteq l_{\infty}^{N}$ be an $n$-dimensional subspace. By Proposition 2.2 of [KT2], the left side of (2.1) is bounded by $\varphi(n, T)$ since $\lambda\left(X_{n}, l_{\infty}^{N}\right) \leq \varphi(n, T)$ is proved there using a duality argument. The supremum in $\varphi(n, T)$ is attained (see Section 4 of [KT2]), say by a probability measure $\mu^{\circ}=\left(\mu_{s}^{\circ}\right)_{s=1}^{N}$ and a $\mu$-orthonormal system $f_{1}^{\circ}, \ldots, f_{n}^{\circ} \in \mathbb{K}^{N}$. Let $f^{\circ}:=\left(\sum_{j=1}^{n}\left|f_{j}\right|^{2}\right)^{1 / 2} \in \mathbb{K}^{N}$ denote the square function. It is shown in Proposition 3.1 of [KT2] by use of Lagrange multipliers that the square function $f^{\circ}$ is constant $\mu$-a.e.; then from the orthonormality, $f^{\circ}(s)=\sqrt{n}$ if $\mu_{s}^{\circ} \neq 0$. (If $\mu_{s}^{\circ}=0$, nothing can be said about $f^{\circ}(s)$; in general $f^{\circ}(s)$ may be non-zero, contrary to what is stated in [KT2].) The proof there relies on an analogue of Lemma 6 derived there under an additional assumption. The crucial point where this is needed is (3.28) of [KT2]. The notation used there is $z_{s k}:=f_{k}^{\circ}(s) \sqrt{\mu_{s}}$. The Lagrange equations of the first kind yield an eigenvalue equation for the map

$$
u:=\left(\operatorname{sgn}\left(\sum_{k=1}^{n} f_{k}^{\circ}(s) \overline{f_{k}^{\circ}(t)}\right) \mu_{t}^{\circ}\right)_{s, t \in T}: \mathbb{K}^{N} \rightarrow \mathbb{K}^{n}
$$

of the form $\mu^{\circ}(s)\left(u f_{k}^{\circ}(s)-\alpha_{k} f_{k}^{\circ}(s)\right)=0$ for $k=1, \ldots, n, s \in T$ (which is a reformulation of (3.16) in [KT2]). Thus with $S:=\operatorname{supp} \mu^{\circ},\left(u f_{k}^{\circ}\right)(s)=$ $\alpha_{k} f_{k}^{\circ}(s)$ for $s \in S$. By (3.27) and the next two lines of [KT2], for each $1 \leq l, m \leq n$ and $s \in T$ one has

$$
0=\left(\alpha_{m}-\alpha_{l}\right) z_{s m} z_{s l}=\left(\alpha_{m}-\alpha_{l}\right) f_{m}^{\circ}(s) f_{l}^{\circ}(s) \mu_{s}^{\circ} .
$$

By Lemma 6 there exists a sequence $l=l_{0}, l_{1}, \ldots, l_{k}=m$ with

$$
\operatorname{supp} f_{l_{r-1}}^{\circ} \cap \operatorname{supp} f_{l_{r}}^{\circ} \cap \operatorname{supp} \mu^{\circ} \neq \emptyset
$$

for all $1 \leq r \leq k$. Thus (2.6) implies that $\alpha_{l}=\alpha_{l_{0}}=\alpha_{l_{1}}=\ldots=\alpha_{l_{k}}=\alpha_{m}$. Hence all values $\alpha_{k}$ coincide, $\alpha_{1}=\ldots=\alpha_{n}=: \alpha$; this is (3.28) of [KT2]. 
The second Lagrange equation (3.15) in [KT2] means that

$$
\sum_{t \in T}\left|\sum_{k=1}^{n} f_{k}^{\circ}(s) \overline{f_{k}^{\circ}(t)}\right| \mu_{t}^{\circ}=\varphi(n, T)=\Lambda
$$

is constant in $s \in S$. Multiplying $u f_{k}^{\circ}=\alpha f_{k}^{\circ}$ pointwise by $\overline{f_{k}^{\circ}}$ and summing over $k=1, \ldots, n$, one deduces from (2.5) and (2.7), using $z \operatorname{sgn} z=|z|$ for $z=\sum_{k=1}^{n} f_{k}^{\circ}(s) \overline{f_{k}^{\circ}(t)}$, that for $s \in S$,

$$
\begin{aligned}
\alpha f^{\circ}(s)^{2} & =\alpha \sum_{k=1}^{n}\left|f_{k}^{\circ}(s)\right|^{2}=\alpha \sum_{k=1}^{n} u f_{k}^{\circ}(s) \cdot \overline{f_{k}^{\circ}(s)} \\
& =\sum_{t \in T}\left|\sum_{k=1}^{n} f_{k}^{\circ}(s) \overline{f_{k}^{\circ}(t)}\right| \mu_{t}^{\circ}=\Lambda .
\end{aligned}
$$

Hence the square function $f^{\circ}$ is constant $\mu^{\circ}$-a.e., $f^{\circ}(s)=\sqrt{\Lambda / \alpha}$ for $s \in S$. Since the $f_{k}$ 's were orthonormal, $f^{\circ}(s)=\sqrt{n}$ for $s \in S$. Hence $\alpha=\Lambda / n$ and $u f_{k}=\Lambda / n \cdot f_{k}$ for $k=1, \ldots, n$. Introducing $x_{t}=n^{-1 / 2}\left(f_{k}^{\circ}(t)\right)_{k=1}^{n} \in \mathbb{K}^{n}$, we have $x_{t} \in S^{n-1}(\mathbb{K})$ and for any $s \in S$,

$$
\varphi(n, T)=n \sum_{t \in T}\left|\left\langle x_{s}, x_{t}\right\rangle\right| \mu_{t}^{\circ}=n \sum_{s, t \in T}\left|\left\langle x_{s}, x_{t}\right\rangle\right| \mu_{t}^{\circ} \mu_{s}^{\circ} .
$$

The vectors $x_{s}$ satisfy (2.2) since the $f_{k}^{\circ}$ are $\mu$-orthonormal. This proves " $\leq$ " in (2.1).

As for the reverse inequality, the Lagrange multiplier approach outlined above (with details in [KT2]) yields a sequence of points $x_{s} \in S^{n-1}(\mathbb{K})$ and a probability measure $\mu$ with (2.2) and

$$
\Lambda=n \sum_{s, t \in T}\left|\left\langle x_{s}, x_{t}\right\rangle\right| \mu_{s} \mu_{t}
$$

and an operator $u$ similar to (2.5), with $S:=\operatorname{supp} \mu, M=|S| \leq N$,

$$
u=\left(\operatorname{sgn}\left(\left\langle x_{s}, x_{t}\right\rangle\right) \mu_{t}\right)_{s, t \in S} \cdot \mathbb{K}^{M} \rightarrow \mathbb{K}^{M}
$$

with $\left(u f_{k}\right)(s)=(\Lambda / n) f_{k}(s)$ for $k=1, \ldots, n, s \in S$. Consider the space $X_{n}$ spanned by the vectors $\left(f_{k}(s)\right)_{s \in S} \subseteq l_{\infty}^{M}$ in $l_{\infty}^{M}$. Then $\left.u\right|_{X_{n}}=(\Lambda / n) \operatorname{Id}_{X_{n}}$, and for any projection $P: l_{\infty}^{N} \rightarrow X_{n}$,

$$
\Lambda=\operatorname{tr}\left(\left.u\right|_{X_{n}}\right) \leq \nu\left(\left.u\right|_{X_{n}}\right) \leq\|P\| \nu(u)=\|P\|
$$

since $\nu(u)=\sum_{t=1}^{N} \mu_{t}=1$. Hence $\Lambda \leq \lambda\left(X_{n}, l_{\infty}^{N}\right)$, which proves " $\geq$ " in (2.1).

Proof of Theorem 1. Assume that $X_{n} \subseteq l_{\infty}^{N}$ has maximal projection constant among $n$-dimensional subspaces of $N$-dimensional superspaces. By Proposition 5, we find points $x_{s} \in S^{n-1}$ and $\mu_{s} \geq 0, s=1, \ldots, N$, with 
$\sum_{s=1}^{N} \mu_{s}=1$ satisfying $(2.2)$ such that

$$
\lambda\left(X_{n}\right)=\lambda\left(X_{n}, l_{\infty}^{N}\right)=\Lambda=\sum_{t=1}^{N} \mu_{t}\left|\left\langle x_{s}, x_{t}\right\rangle\right|, \quad s \in S:=\operatorname{supp} \mu .
$$

Let $M:=|S| \leq N$. By Proposition 5, too, suppose $u=\left(\operatorname{sgn}\left(\left\langle x_{s}, x_{t}\right\rangle\right) \mu_{t}\right)_{s, t \in S}$ : $l_{\infty}^{M} \rightarrow l_{\infty}^{M}$ maps $\widetilde{X}_{n}$ into itself where $\widetilde{X}_{n}=\operatorname{span}\left(f_{1}, \ldots, f_{n}\right), f_{j}=\left(x_{s j}\right)_{s \in S} \in$ $\mathbb{K}^{M}$ for $j=1, \ldots, n$, and in fact $\left.u\right|_{\widetilde{X}_{n}}=(\Lambda / n) \operatorname{Id}_{\widetilde{X}_{n}}$. (If $\operatorname{supp} \mu=\{1, \ldots, N\}$, we may take $X_{n}=\widetilde{X}_{n}$.) Hence $\operatorname{tr}\left(\left.u\right|_{\widetilde{X}_{n}}\right)=\Lambda$ and $\nu(u)=\sum_{t \in S} \sup _{s}\left|u_{s t}\right|=$ $\sum_{t \in S} \mu_{s}=1$. For any projection $Q: l_{\infty}^{M} \rightarrow l_{\infty}^{M}$ onto $\widetilde{X}_{n}$,

$$
\Lambda=\operatorname{tr}\left(\left.u\right|_{\widetilde{X}_{n}}\right) \leq \nu\left(\left.u\right|_{\widetilde{X}_{n}}\right) \leq\|Q\| \nu(u)=\|Q\|,
$$

with the $l_{2}^{M}(\mu)$-orthogonal projection $P$ attaining $\|P\|=\Lambda$.

Consider now $\widetilde{X}_{n}$ as a subspace of $l_{1}^{M}(\mu)$, i.e. $\mathbb{K}^{M}$ equipped with the norm given by $\|x\|_{1, \mu}=\sum_{t \in S} \mu_{t}\left|\left\langle x, x_{t}\right\rangle\right|$, and denote this space by $Y_{n}$. Let $D_{\mu}: l_{1}^{M}(\mu) \rightarrow l_{1}^{M}$ be the diagonal map $\left(y_{s}\right) \mapsto\left(\mu_{s} y_{s}\right)$; it is an isometry. The map

$$
l_{1}^{M}(\mu) \stackrel{D_{\mu}}{\longrightarrow} l_{1}^{M} \stackrel{u^{*}}{\longrightarrow} l_{1}^{M} \stackrel{D_{\mu}^{-1}}{\longrightarrow} l_{1}^{M}(\mu)
$$

has as its matrix representation $\left(\mu_{s}^{-1} u_{t s} \mu_{t}\right)_{s, t \in S}=\left(u_{s t}\right)_{s, t \in S}=u$ since $u_{s t}=\operatorname{sgn}\left(\left\langle x_{s}, x_{t}\right\rangle\right) \mu_{t}$. Since $\nu\left(u^{*}\right)_{l_{1}^{M}}=\nu(u)_{l_{\infty}^{M}}=1$ and $D_{\mu}$ is an isometry, $\nu\left(u: l_{1}^{M}(\mu) \rightarrow l_{1}^{M}(\mu)\right)=1$. Hence as above, for any projection $Q: l_{1}^{M}(\mu) \rightarrow$ $l_{1}^{M}(\mu)$ onto $Y_{n}$,

$$
\Lambda=\operatorname{tr}\left(\left.u\right|_{Y_{n}}\right) \leq \nu\left(\left.u\right|_{Y_{n}}\right) \leq\|Q\| \nu(u)=\|Q\| .
$$

Thus $\lambda\left(Y_{n}, l_{1}^{M}(\mu)\right) \geq \Lambda=\lambda\left(X_{n}, l_{\infty}^{N}\right)$. But $\lambda\left(X_{n}\right)$ was maximal among $n$ dimensional subspaces of $N$ - (hence also for $M$-) dimensional superspaces. Hence $\lambda\left(Y_{n}, l_{1}^{M}(\mu)\right) \leq \Lambda$ holds as well. Taking $Z_{n}=D_{\mu}\left(Y_{n}\right) \subseteq l_{1}^{M}$, we can also realize such a space as a subspace of $l_{1}^{M}, \lambda\left(Z_{n}, l_{1}^{M}\right)=\lambda\left(\widetilde{X}_{n}, l_{\infty}^{M}\right)=$ $\lambda\left(X_{n}\right)=\Lambda$.

In general, $X_{n}$ will not be isometric to $Y_{n}$ or $Z_{n}$ except for $\mathbb{K}=\mathbb{R}, n=2$ when these spaces have the regular hexagon as their unit ball. Now let us consider the three-dimensional real case.

Proof of Proposition 2. For $\mathbb{K}=\mathbb{R}, n=3$ we have $G(3)=(1+\sqrt{5}) / 2$ $=: \Phi$. The space $X_{3} \subseteq l_{\infty}^{6}$ having as its unit ball the dodecahedron attains this bound, $\lambda\left(X_{3}\right)=\Phi$; cf. [KT2]. The dual unit ball, the icosahedron, is the convex hull of its six equiangular diagonals $x_{1}, \ldots, x_{6} \in S^{2} \subset \mathbb{R}^{3}$ given by the vectors

$$
c\left(\begin{array}{c}
\phi \\
\pm 1 \\
0
\end{array}\right), c\left(\begin{array}{c}
0 \\
\phi \\
\pm 1
\end{array}\right), c\left(\begin{array}{c} 
\pm 1 \\
0 \\
\phi
\end{array}\right), \quad c:=\frac{1}{\sqrt{\phi+2}}
$$


with $\left|\left\langle x_{s}, x_{t}\right\rangle\right|=1 / \sqrt{5}$ for $1 \leq s, t \leq 6$. Thus $\|x\|_{X_{3}}=\sup _{1 \leq s \leq 6}\left|\left\langle x, x_{s}\right\rangle\right|$. Take $\mu_{s}=1 / 6$ for $s=1, \ldots, 6$. Then $3 \cdot \sum_{t=1}^{6} \mu_{t}\left|\left\langle x_{s}, x_{t}\right\rangle\right|=\phi$; this attains the sup in (2.1), (2.2) being satisfied. The map $x \mapsto\left(\left\langle x, x_{s}\right\rangle\right)_{s=1}^{6}$ realizes the isometric imbedding $X_{3} \hookrightarrow l_{\infty}^{6}$. The "extremal" map $u: l_{\infty}^{N} \rightarrow l_{\infty}^{N}$ used in the proof of Theorem 1 is in this case

$$
\left.u=\left(\operatorname{sgn}\left\langle x_{s}, x_{t}\right\rangle\right) \mu_{t}\right)_{s, t=1}^{6}: l_{\infty}^{6} \rightarrow l_{\infty}^{6} .
$$

By the same proof of Theorem 1, the same linear space, but considered as a subspace of $l_{1}^{6}$, denoted by $Y_{3} \subseteq l_{1}^{6}$, has the same projection constant: $\lambda\left(Y_{3}\right)=\lambda\left(Y_{3}, l_{1}^{6}\right)=\phi$. This holds since $u$ is symmetric and $D_{\mu}=\frac{1}{6} \operatorname{Id}_{6}$. However, the unit ball of $Y_{3}$ is not the dodecahedron; it has 12 regular pentagons and 20 regular triangles as its faces and thus is not isometric to $X_{3}$ : The norm on $Y_{3}$ is given by $\|x\|_{Y_{3}}=\frac{1}{6} \sum_{s=1}^{6}\left|\left\langle x, x_{s}\right\rangle\right|$; writing it as

$$
\|x\|_{Y_{3}}=\frac{1}{6} \sup _{\varepsilon_{s}= \pm 1}\left|\left\langle x, \sum_{s=1}^{6} \varepsilon_{s} x_{s}\right\rangle\right|
$$

one finds that only 16 combinations $\left(\varepsilon_{1}, \ldots, \varepsilon_{6}\right)$ of signs are needed to represent the norm and thus $Y_{3}$ is isometrically imbeddable into $l_{\infty}^{16}$. The choice $\varepsilon_{s}=\operatorname{sgn}\left(\left\langle x_{s}, x_{t}\right\rangle\right)=6 u_{s t}$ for fixed $t$ yields

$$
\frac{1}{6} \sum_{s=1}^{6} \varepsilon_{s} x_{s}=\frac{\phi}{3} x_{s} \quad(s=1, \ldots, 6) .
$$

Thus, eliminating the factor $\phi / 3$, the points $x_{1}, \ldots, x_{6}$ are again needed to imbed $Y_{3}$ into $l_{\infty}$; in addition, one needs the 10 vectors (also after multiplying by $3 / \phi$ )

$$
c\left(\begin{array}{c}
\phi \\
0 \\
\pm \phi^{-1}
\end{array}\right), c\left(\begin{array}{c} 
\pm \phi^{-1} \\
\phi \\
0
\end{array}\right), c\left(\begin{array}{c}
0 \\
\pm \phi^{-1} \\
\phi
\end{array}\right), c\left(\begin{array}{c}
1 \\
\pm 1 \\
\pm 1
\end{array}\right), \quad c:=\frac{1}{\sqrt{\phi+2}} .
$$

If these 16 vectors are called $x_{1}, \ldots, x_{16}$, one has

$$
\frac{3}{\phi}\|x\|_{Y_{3}}=\sup _{1 \leq s \leq 16}\left|\left\langle x, x_{s}\right\rangle\right|, \quad Y_{3} \hookrightarrow l_{\infty}^{16} \text { isometrically. }
$$

We remark that $x_{7}, \ldots, x_{16}$ are one half of the vertices of the regular dodecahedron and thus the unit ball of $Y_{3}$ is the intersection of the dodecahedron and a multiple of the icosahedron yielding the above-mentioned face structure. In fact, if the vertices of the icosahedron are chosen to be the midpoints of the faces of the dodecahedron, one has to multiply this icosahedron by $\phi$ and intersect it with the dodecahedron to get the unit ball of $Y_{3}$. 
In the case of complex 2-dimensional spaces, there are four equiangular vectors

$$
\left(\begin{array}{l}
1 \\
0
\end{array}\right), \frac{1}{\sqrt{3}}\left(\begin{array}{c}
1 \\
\sqrt{2} w^{j}
\end{array}\right), \quad j=0,1,2, w=\exp (2 \pi i / 3),
$$

in $\mathbb{C}^{2}$. If we call them $z_{1}, \ldots, z_{4} \in \mathbb{C}^{2}$, then the space with norm

$$
\|z\|_{X_{2}}=\sup _{1 \leq s \leq 4}\left|\left\langle z, z_{s}\right\rangle\right|
$$

has maximal projection constant among 2-dimensional complex spaces with $\lambda\left(X_{2}\right)=(1+\sqrt{3}) / 2, X_{2} \subseteq l_{\infty}^{4}$ (cf. [KT2]). As a subspace $Y_{2}$ of $l_{1}^{4}$, it also has $\lambda\left(Y_{2}\right)=\lambda\left(Y_{2}, l_{1}^{4}\right)=(1+\sqrt{3}) / 2$, but is not isometric to $X_{2}$ since the Banach-Mazur distances to Hilbert space satisfy

$$
d\left(X_{2}, l_{2}^{2}\right)=\sqrt{3 / 2} \neq d\left(Y_{2}, l_{2}^{2}\right)=(1+\sqrt{3}) / \sqrt{6} .
$$

The distance ellipsoid here is the standard euclidean ball in $\mathbb{C}^{2}$ by symmetry reasons, the values of $\sqrt{3 / 2}$ and $(1+\sqrt{3}) / \sqrt{6}$ are obtained by calculating the maximum and minimum of $\|z\|_{2}$ subject to $\|z\|_{X_{2}}=1$ or $\|z\|_{Y_{2}}=1$; the quotient of these maxima and minima then gives the above distance values. The maxima and minima are attained at 4 points each, up to factors $e^{i \theta}$. We would like to thank Prof. A. Pełczyński for some stimulating discussions on this topic.

REMARK. The fact that the space $Y_{3}$, imbedded into $l_{\infty}^{16}$, allows no projection of norm $<\phi$, can also be checked by a map $\widetilde{u}: l_{\infty}^{16} \rightarrow l_{\infty}^{16}$ similar to $u$ for $X_{3} \subseteq l_{\infty}^{6}$. One may just take

$$
\widetilde{u}=\left(\operatorname{sgn}\left\langle x_{s}, x_{t}\right\rangle \mu_{t}\right)_{s, t=1}^{16}: l_{\infty}^{16} \rightarrow l_{\infty}^{16}
$$

where $\left(x_{s}\right)_{s=1}^{16} \subset \mathbb{R}^{3}$ are the 16 vectors given in the previous proof and where $\mu_{t}=0$ for all $t=7, \ldots, 16$. Again $\nu(\widetilde{u})=1$ and $\left.\widetilde{u}\right|_{Y_{3}}=(\phi / 3) \operatorname{Id}_{Y_{3}}$. Thus $\widetilde{u}$ has 10 columns of zeros; for the rows $s=7, \ldots, 16$ Proposition 5 gives no information on the square function $\left\|x_{s}\right\|_{2}^{2}$ as compared to $\left\|x_{s}\right\|_{2}^{2}=1$ for $s=1, \ldots, 6$. In fact, $\left\|x_{s}\right\|_{2}^{2}=3 /(\phi+2)<1$ for $s=7, \ldots, 16$.

Proof of Theorem 3. Let $X_{n} \subseteq l_{\infty}^{N}$ be an $n$-dimensional space with maximal projection constant for $n$-dimensional spaces, $\lambda\left(X_{n}\right)=g(n)$, and where $N \leq e^{\sqrt{n} /(8 e)}$ holds. By Proposition 5, we conclude that there are unit vectors $\left(x_{s}\right)_{s=1}^{N}$ in $l_{2}^{n}, x_{s} \in S^{n-1}$, and a probability measure $\mu=\left(\mu_{s}\right)_{s=1}^{N}$ on $\{1, \ldots, N\}$ such that:

- Using (1.4) we identify $X_{n}$ with the space $\mathbb{K}^{n}$, equipped with the norm $\|x\|=\sup _{1 \leq s \leq N}\left|\left\langle x, x_{s}\right\rangle\right|$. Then the dual unit ball is the absolutely convex hull of the vectors $x_{s}$.

- $\Lambda:=\lambda\left(X_{n}\right)=n \sum_{t=1}^{N} \mu_{t}\left|\left\langle x_{s}, x_{t}\right\rangle\right|$ for all $s=1, \ldots, N$.

- $\operatorname{Id}_{n}=n \sum_{t=1}^{N} \mu_{t}\left\langle\cdot, x_{t}\right\rangle x_{t}$ on $l_{2}^{n}$.

- $\left.u\right|_{X_{n}}=(\Lambda / n) \operatorname{Id}_{n}$, where $u=\left(\operatorname{sgn}\left(\left\langle x_{s}, x_{t}\right\rangle\right) \mu_{t}\right)_{s, t=1}^{N}: l_{\infty}^{N} \rightarrow l_{\infty}^{N}$. 
We will assume for simplicity and without loss of generality that all $\mu_{s}$ are $>0$. Thus $X_{n}$ in $l_{\infty}^{N}$ is spanned by the vectors $f_{j}:=\left(x_{s j}\right)_{s=1}^{N}$ which are, up to the factor $\sqrt{n}$, orthonormal vectors in $l_{2}^{N}(\mu)$. Since $\operatorname{tr}\left(\left.u\right|_{X_{n}}\right)=\Lambda$ and the nuclear norm of $u$ in $l_{\infty}^{N}$ is $1, \nu(u)=\sum_{t=1}^{N} \mu_{t}=1$, any projection $P: l_{\infty}^{N} \rightarrow X_{n}$ must have norm $\geq \Lambda$,

$$
\Lambda=\operatorname{tr}\left(\left.u\right|_{X_{n}}\right) \leq \nu\left(\left.u\right|_{X_{n}}\right) \leq\|P\| \nu(u)=\|P\| .
$$

We will construct a vector $x_{N+1}$ which is not in the absolutely convex hull of $x_{1}, \ldots, x_{N}$ such that the map $x \mapsto\left(\left\langle x, x_{s}\right\rangle\right)_{s=1}^{N+1}$ yields another $n$-dimensional extremal space $Y_{n} \subseteq l_{\infty}^{N+1}, \lambda\left(X_{n}\right)=\lambda\left(Y_{n}\right)$, which is not isometric to $X_{n}$ since the unit ball of $Y_{n}$ has more faces than the one of $X_{n}$.

Let $\alpha:=1 /(2 \sqrt{\log N})$. For any $y \in S^{n-1}$ and $t \in\{1, \ldots, N\}$ let

$$
\varepsilon_{t}(y):=\sqrt{n} \alpha\left\langle y, x_{t}\right\rangle \text {. }
$$

Then

$$
z(y):=\frac{n}{\Lambda} \sum_{t=1}^{N} \varepsilon_{t}(y) \mu_{t} x_{t}=\frac{\sqrt{n}}{\Lambda} \alpha n \sum_{t=1}^{N} \mu_{t}\left\langle y, x_{t}\right\rangle x_{t}=\frac{\sqrt{n}}{\Lambda} \alpha y,
$$

so $\|z(y)\|_{2}=\sqrt{n} \alpha / \Lambda$. We estimate the average norm of $z(y)$ in $X_{n}$. For this, let $m$ be the normalized Lebesgue measure on $S^{n-1}$. Take $p=2$. Then

$$
\begin{aligned}
\int_{S^{n-1}} & \|z(y)\|_{X_{n}} d m(y)=\int_{S^{n-1}} \sup _{1 \leq s \leq N}\left|\left\langle z(y), x_{s}\right\rangle\right| d m(y) \\
\leq & \int_{S^{n-1}}\left(\sum_{s=1}^{N}\left|\left\langle z(y), x_{s}\right\rangle\right|^{p}\right)^{1 / p} d m(y) \leq\left(\sum_{s=1}^{N} \int_{S^{n-1}}\left|\left\langle z(y), x_{s}\right\rangle\right|^{p} d m(y)\right)^{1 / p} \\
\leq & N^{1 / p}\|z(y)\|_{2}\left(\int_{S^{n-1}}\left|y_{1}\right|^{p} d m(y)\right)^{1 / p} \leq N^{1 / p} \frac{\sqrt{n}}{\Lambda} \alpha \sqrt{\frac{p}{n}}
\end{aligned}
$$

Here we used the generalized triangle inequality and the rotation invariance of $m$. The moments $\left(\int_{S^{n-1}}\left|y_{1}\right|^{p} d m(y)\right)^{1 / p}$ are explicitly known in terms of Gamma functions: they can be estimated by $\sqrt{p / n}$ for $p>2$. Choosing $p=\log N$, by definition of $\alpha$ we get

$$
\int_{S^{n-1}}\|z(y)\|_{X_{n}} d m(y) \leq \frac{e}{\Lambda} .
$$

By Chebyshev's inequality,

$$
m\left\{y \in S^{n-1} \mid\|z(y)\|_{X_{n}}<2 \frac{e}{\Lambda}\right\}>\frac{1}{2} .
$$

Since $2 e / \Lambda<n / \Lambda^{2} \alpha^{2}$ by assumption on $N$, for these vectors $z(y)$ one has

$$
\|z(y)\|_{X_{n}}<\frac{2 e}{\Lambda}<\frac{n}{\Lambda^{2}} \alpha^{2}=\|z(y)\|_{2}^{2} \leq\|z(y)\|_{X_{n}}\|z(y)\|_{X_{n}^{*}} .
$$


Hence $\|z(y)\|_{X_{n}^{*}}>1$, which means that $z(y)$ is not in the absolutely convex hull of the vectors $x_{1}, \ldots, x_{N}$. On the other hand, we want to guarantee that the values $\varepsilon_{t}(y)$ can be bounded by 1 uniformly in $t$. Integration by polar coordinates yields the following well known tail estimate for linear functionals ( $t$ being fixed):

$$
\begin{aligned}
m\left\{y \in S^{n-1}(\mathbb{K})||\left\langle y, x_{t}\right\rangle \mid>\beta\right\} \\
= \begin{cases}\int_{\beta}^{1}\left(1-u^{2}\right)^{(n-3) / 2} d u / \int_{0}^{1}\left(1-u^{2}\right)^{(n-3) / 2} d u \leq e^{-n \beta^{2} / 2} \quad(\mathbb{K}=\mathbb{R}), \\
\int_{\beta}^{1} u\left(1-u^{2}\right)^{n-2} d u / \int_{0}^{1} u\left(1-u^{2}\right)^{n-2} d u \\
=\left(1-\beta^{2}\right)^{n-1} \leq e^{-n \beta^{2} / 2} \quad(\mathbb{K}=\mathbb{C})\end{cases}
\end{aligned}
$$

for $n \geq 2,0<\beta \leq 1$. (For integration in the complex case, $\mathbb{C}^{n}$ is identified with $\mathbb{R}^{2 n}$.) Choosing $\beta=1 /(\alpha \sqrt{n})$, we get

$$
m\left\{y \in S^{n-1}||\left\langle y, x_{t}\right\rangle \mid>1 /(\alpha \sqrt{n})\right\} \leq e^{-\alpha^{-2} / 2} \leq 1 / N^{2}<1 /(2 N) .
$$

Letting $t$ vary from 1 to $N$, one finds for the complement

$$
m\left\{y \in S^{n-1}||\left\langle y, x_{t}\right\rangle \mid \leq 1 /(\alpha \sqrt{n}) \text { for all } t=1, \ldots, N\right\}>1 / 2 .
$$

By (2.8) and (2.9) we can find a vector $\bar{y} \in S^{n-1}$ such that

- $\|z(\bar{y})\|_{X_{n}}<2 e / \Lambda$, implying $\|z(\bar{y})\|_{X_{n}^{*}}>1$.

- $\left|\varepsilon_{t}(\bar{y})\right|=\sqrt{n} \alpha\left|\left\langle\bar{y}, x_{t}\right\rangle\right| \leq 1$ for all $t=1, \ldots, N$.

Put $x_{N+1}=z(\bar{y})$. Then $\left\|x_{N+1}\right\|_{2}=\sqrt{n} \alpha / \Lambda \leq 2 \alpha<1(\Lambda$ is close to $\sqrt{n})$ and $x_{N+1} \notin$ absolutely convex hull of $\left(x_{1}, \ldots, x_{N}\right)$. Define $Y_{n}$ by $\|x\|_{Y_{n}}:=$ $\sup _{1 \leq s \leq N+1}\left|\left\langle x, x_{s}\right\rangle\right|, x \in \mathbb{K}^{n}$, and let $\mu_{N+1}=0$. Then $\|x\|_{Y_{n}} \geq\|x\|_{X_{n}}$ and there are points $x \in \mathbb{K}^{n}$ with $\|x\|_{Y_{n}}>\|x\|_{X_{n}}$. The unit ball of $Y_{n}$ thus has more faces than $X_{n}$, and $Y_{n}$ is not isometric to $X_{n}$. Let $\widetilde{f}_{j}=\left(x_{s j}\right)_{s=1}^{N+1}$. Then $Y_{n}=\operatorname{span}\left(\widetilde{f}_{1}, \ldots, \widetilde{f}_{n}\right) \subseteq l_{\infty}^{N+1}$; these vectors are homothetic to an orthonormal basis in $Y_{n}$ as a subspace of $l_{2}^{N+1}(\mu)$. We define an extension $\widetilde{u}: l_{\infty}^{N+1} \rightarrow l_{\infty}^{N+1}$ of the map $u: l_{\infty}^{N} \rightarrow l_{\infty}^{N}$ by putting $\widetilde{u}_{s t}=u_{s t}$ if $1 \leq s, t \leq N$, $\widetilde{u}_{s, N+1}=0$ for $s=1, \ldots, N+1$ and $\widetilde{u}_{N+1, t}=\varepsilon_{t}(\bar{y}) \mu_{t}$ for $t=1, \ldots, N$. Then $\left(\widetilde{u} \tilde{f}_{j}\right)_{s}=\left(u f_{j}\right)_{s}=(\Lambda / n)\left(f_{j}\right)_{s}=(\Lambda / n)\left(\tilde{f}_{j}\right)_{s}$ for $s=1, \ldots, N$, and by definition of $z(\bar{y})$,

$$
\begin{aligned}
\left(\widetilde{u} \widetilde{f}_{j}\right)_{N+1} & =\sum_{t+1}^{N} \widetilde{u}_{N+1, t}\left(\widetilde{f}_{j}\right)_{t}=\sum_{t=1}^{N} \varepsilon_{t}(\bar{y}) \mu_{t} x_{t j} \\
& =\frac{\Lambda}{n} z(\bar{y})_{j}=\frac{\Lambda}{n} x_{N+1, j}=\frac{\Lambda}{n}\left(\widetilde{f}_{j}\right)_{N+1} .
\end{aligned}
$$

We thus found that $\left.\widetilde{u}\right|_{Y_{n}}=(\Lambda / n) \operatorname{Id}_{Y_{n}}$. The nuclear norm of $\widetilde{u}: l_{\infty}^{N+1} \rightarrow l_{\infty}^{N+1}$ 
is (in view of $\mu_{n+1}=0$ )

$$
\nu(\widetilde{u})=\sum_{t=1}^{N+1} \sup _{1 \leq s \leq N+1}\left|\widetilde{u}_{s t}\right|=\sum_{t=1}^{N} \mu_{t}=1 .
$$

If $P: l_{\infty}^{N+1} \rightarrow Y_{n}$ is any projection,

$$
\Lambda=\operatorname{tr}\left(\left.\widetilde{u}\right|_{Y_{n}}\right) \leq \nu\left(\left.\widetilde{u}\right|_{Y_{n}}\right) \leq\|P\| \nu(\widetilde{u})=\|P\| .
$$

Thus $\Lambda\left(Y_{n}\right) \geq \Lambda=\lambda\left(X_{n}\right)$. However, $\lambda\left(X_{n}\right)$ was maximal among all $n$ dimensional spaces. Hence $\lambda\left(Y_{n}\right)=\lambda\left(X_{n}\right)=\Lambda$ and $Y_{n}$ is not isometric to $X_{n}$. Obviously, the construction yields infinitely many non-isometric spaces with maximal projection constant $\Lambda$.

Proof of Proposition 4. (a) As subspaces of $l_{\infty}^{N}$, these spaces have already been considered in [KT1]. For $n=p^{m}+1$ there exist numbers $d_{1}, \ldots, d_{n} \in$ $\{0, \ldots, N-1\}$ such that the differences $d_{i}-d_{j}$ modulo $N$ are all different and yield all $n(n-1)=N-1$ integers between 1 and $N-1$; see [HR]. Define $x_{s}:=n^{-1 / 2}\left(\exp \left((2 \pi i / N) d_{j} s\right)\right)_{j=1}^{n} \in S^{n-1}(\mathbb{C})$ for $s=1, \ldots, N$ and let $f_{j}=\left(x_{s j}\right)_{s=1}^{N} \in \mathbb{C}^{n}$. Then

$$
\left\langle f_{j}, f_{k}\right\rangle=\sum_{s=1}^{N} \exp \left(\frac{2 \pi i}{N}\left(d_{j}-d_{k}\right) s\right) / n=(N / n) \delta_{j k}
$$

and hence

$$
\operatorname{Id}_{n}=\frac{n}{N} \sum_{s=1}^{N}\left\langle\cdot, x_{s}\right\rangle x_{s} \quad\left(\text { on } \mathbb{C}^{n}\right)
$$

The vectors $\left(x_{s}\right)_{s=1}^{N}$ are equiangular as the evaluation of $\left|\left\langle x_{s}, x_{t}\right\rangle\right|^{2}$ shows (see $[\mathrm{KT} 1])$. One finds

$$
\left|\left\langle x_{s}, x_{t}\right\rangle\right|=\sqrt{n-1} / n \quad \text { for } 1 \leq s \neq t \leq N .
$$

Let $Z_{n}=\operatorname{span}\left(f_{1}, \ldots, f_{n}\right) \subseteq \mathbb{C}^{N}$. As a subspace of $l_{2}^{N},\left(\sqrt{n / N} f_{j}\right)_{j=1}^{n}$ is an orthonormal basis in $Z_{n}, \operatorname{dim} Z_{n}=n$, and $P:=(n / N)\left(\left\langle x_{s}, x_{t}\right\rangle\right)_{s, t=1}^{n}$ is a projection onto $Z_{n}$ (the orthogonal projection in $l_{2}^{N}$ ).

Let $X_{n}$ and $Y_{n}$ denote the linear space $Z_{n}$ considered as a subspace of $l_{\infty}^{N}$ and $l_{1}^{N}$, respectively. Then

$$
\lambda\left(Y_{n}\right) \leq\|P\|=\frac{n}{N} \sup _{t} \sum_{s=1}^{N}\left|p_{s t}\right|=\frac{n}{N}\left(1+(N-1) \frac{\sqrt{n-1}}{n}\right)=F(n, N) .
$$

The last equality is verified by calculation ( $F$ is given in Section 1$)$. Similarly $\lambda\left(X_{n}\right) \leq F(n, N)$ since $P$ is hermitean.

Let

$$
u:=\left(\left\langle x_{s}, x_{t}\right\rangle\right)_{s, t=1}^{N}-\left(1-\frac{\sqrt{n-1}}{n}\right) \operatorname{Id}_{N}: \mathbb{C}^{N} \rightarrow \mathbb{C}^{N}
$$


Then for all $1 \leq s, t \leq N$,

$$
\left|u_{s t}\right|=\frac{\sqrt{n-1}}{n} \text { and }\left.u\right|_{Z_{n}}=\left(\frac{N}{n}-1+\frac{\sqrt{n-1}}{n}\right) \operatorname{Id}_{Z_{n}} .
$$

Hence the trace of $u$ on $Z_{n}$ is $\operatorname{tr}\left(\left.u\right|_{Z_{n}}\right)=(N-n+\sqrt{n-1})$. On the other hand, the nuclear norm of the hermitean map $u$, considered in either $l_{1}^{N}$ or $l_{\infty}^{N}$, is

$$
\nu(u)=\sum_{s=1}^{N} \sup _{t}\left|u_{s t}\right|=N \frac{\sqrt{n-1}}{n} .
$$

This implies, for any projection $Q: l_{1}^{N} \rightarrow Y_{n}$,

$$
N-n+\sqrt{n-1}=\operatorname{tr}\left(\left.u\right|_{Y_{n}}\right) \leq \nu\left(\left.u\right|_{Y_{n}}\right) \leq\|Q\| \nu(u)=\|Q\| N \frac{\sqrt{n-1}}{n} .
$$

Thus

$$
\lambda\left(Y_{n}\right) \geq \frac{N-n+\sqrt{n-1}}{N} \frac{n}{\sqrt{n-1}}=F(n, N),
$$

the last equality again being the result of a calculation. Similarly $\lambda\left(X_{n}\right) \geq$ $F(n, N)$. We showed that $\lambda\left(X_{n}\right)=\lambda\left(Y_{n}\right)=F(n, N)$, which is the maximal possible value (see $[\mathrm{KLL}]$ ).

(b) We show that the Banach-Mazur distance of $X_{n}$ to $l_{2}^{n}$ is $\sqrt{n}$. (By John's theorem, this is extremal since for any $n$-dimensional space $Z_{n}$ one has $d\left(Z_{n}, l_{2}^{n}\right) \leq \sqrt{n}$. $)$ The proof is similar to the one in [KT1].

Let $\beta_{j}:=\exp \left((2 \pi i / N) d_{j}\right)$ for $j=1, \ldots, n$ and $I: X_{n} \rightarrow X_{n}$ be defined by $\sum_{j=1}^{n} a_{j} f_{j} \mapsto \sum_{j=1}^{n} \beta_{j} a_{j} f_{j}$. Clearly $I$ is an isometry and $I^{N}=\mathrm{Id}$. Any inner product $[\cdot, \cdot]$ on $X_{n}$ which is invariant under $I$ is diagonal in the basis $\left(f_{j}\right)$ of $X_{n}$. In fact, if

$$
[x, y]=\sum_{k, l=1}^{n} t_{k l} a_{k} \bar{b}_{l}, \quad x=\sum_{k=1}^{n} a_{k} f_{k}, y=\sum_{l=1}^{n} b_{l} f_{l},
$$

$[I x, I y]=[x, y]$ for all $x, y \in X_{n}$ implies $t_{k l}=t_{k l} \beta_{k} \bar{\beta}_{l}$. For $k \neq l$, clearly $\beta_{k} \bar{\beta}_{l} \neq 1$, hence $t_{k l}=0$ for $k \neq l$.

Now let $(\cdot, \cdot)$ be an inner product on $X_{n}$ which determines the BanachMazur distance $d=d\left(X_{n}, l_{2}^{n}\right)$, normalized so that

$$
\left(1 / d^{2}\right)\|x\|^{2} \leq(x, x) \leq\|x\|^{2}, \quad x \in X_{n} .
$$

Define the inner product $[\cdot, \cdot]$ by

$$
[x, y]:=\frac{1}{N} \sum_{s=0}^{N-1}\left(I^{s} x, I^{s} y\right), \quad x, y \in X_{n} .
$$

Then $[I x, I y]=[x, y]$, and also

$$
\left(1 / d^{2}\right)\|x\|^{2} \leq[x, x] \leq\|x\|^{2}, \quad x \in X_{n} .
$$


By the preceding remark, there are $\lambda_{1}, \ldots, \lambda_{n} \in \mathbb{C}$ such that

$$
[x, y]=\sum_{k=1}^{n} \lambda_{k} a_{k} \bar{b}_{k}, \quad x=\sum_{n=1}^{n} a_{k} f_{k}, y=\sum_{k=1}^{n} b_{k} f_{k} .
$$

Since $[x, x] \leq\|x\|^{2}, \lambda_{k} \leq\left\|f_{k}\right\|^{2}=1 / n$ for all $k=1, \ldots, n$. Let $z:=\sum_{k=1}^{n} f_{k}$. Then $[z, z]=\sum_{k=1}^{n} \lambda_{k}$ and, taking $s=0$, we find

$$
\|z\|=\sup _{0 \leq s<N} \frac{1}{\sqrt{n}}\left|\sum_{k=1}^{n} \exp \left(\frac{2 \pi i}{N} d_{j} s\right)\right|=\sqrt{n} .
$$

So

$$
d \geq \sup _{x \neq 0}\|x\| /[x, x]^{1 / 2} \geq \frac{\sqrt{n}}{\left(\sum_{k=1}^{n} \lambda_{k}\right)^{1 / 2}} \geq \sqrt{n} .
$$

Since by John's theorem, $d \leq \sqrt{n}$, we find $d=\sqrt{n}$.

As a subspace of $l_{1}^{N}$, the distance of the space $Y_{n}$ to $l_{2}^{n}$, however, is uniformly bounded by $\sqrt{2}$ as we now show. Thus $X_{n}$ and $Y_{n}$ cannot be isometric. In fact, for large $n$, they have very different distances to $l_{2}^{n}$. For $1 \leq p<\infty$ and $x \in \mathbb{C}^{n}$, let

$$
\|x\|_{p}=\left(\frac{1}{N} \sum_{s=1}^{N}\left|\left\langle x, x_{s}\right\rangle\right|^{p}\right)^{1 / p} .
$$

We calculate the 4-norm:

$$
\begin{aligned}
& \sum_{s=1}^{N}\left|\left\langle x, x_{s}\right\rangle\right|^{4} \\
& \quad=\frac{1}{n^{2}} \sum_{j_{1}, j_{2}, j_{3}, j_{4}=1}^{n} x_{j_{1}} \bar{x}_{j_{2}} x_{j_{3}} \bar{x}_{j_{4}} \sum_{s=1}^{N} \exp \left(\frac{2 \pi i}{N}\left(d_{j_{1}}-d_{j_{2}}+d_{j_{3}}-d_{j_{4}}\right) s\right) .
\end{aligned}
$$

Since $\left(d_{i}-d_{j}\right)(N)$ for $i \neq j$ runs over all numbers from 1 to $N-1$ exactly once, $d_{j_{1}}-d_{j_{2}}+d_{j_{3}}-d_{j_{4}}$ is 0 modulo $n$ if and only if either $\left(j_{1}=j_{2}\right.$ and $\left.j_{3}=j_{4}\right)$ or $\left(j_{1}=j_{4}\right.$ and $\left.j_{2}=j_{3}\right)$.

In this case, the inner sum is $N$, else it is 0 . Hence

$$
\begin{aligned}
\|x\|_{4}:= & \left(\frac{1}{N} \sum_{s=1}^{N}\left|\left\langle x, x_{s}\right\rangle\right|^{4}\right)^{1 / 4} \\
= & \frac{1}{\sqrt{n}}\left(2 \sum_{j \neq k=1}^{n}\left|x_{j}\right|^{2}\left|x_{k}\right|^{2}+\sum_{j=1}^{n}\left|x_{j}\right|^{4}\right)^{1 / 4} \\
& \leq \frac{\sqrt[4]{2}}{\sqrt{n}}\left(\sum_{j=1}^{n}\left|x_{j}\right|^{2}\right)^{1 / 2}=\sqrt[4]{2}\|x\|_{2}
\end{aligned}
$$


where the last equality is easy. A standard interpolation argument now yields the distance estimate of $Y_{n}$ to $l_{2}^{n}$ : By Hölder's inequality

$$
\|x\|_{2} \leq\|x\|_{1}^{1 / 3}\|x\|_{4}^{2 / 3} \leq(\sqrt[4]{2})^{2 / 3}\|x\|_{1}^{1 / 3}\|x\|_{2}^{2 / 3},
$$

and thus $\|x\|_{2} \leq \sqrt{2}\|x\|_{1}$. Since trivially $\|x\|_{1} \leq\|x\|_{2}$ holds, we find that $d\left(Y_{n}, l_{2}^{n}\right) \leq \sqrt{2}$

\section{References}

[CL] B. Chalmers and G. Lewicki, Symmetric spaces with maximal projection constant, J. Funct. Anal. 200 (2003), 1-22.

[HR] H. Halberstam and K. F. Roth, Sequences, Springer, New York, 1983.

[KS] M. I. Kadets and M. G. Snobar, Certain functionals on the Minkowski compactum, Math. Notes 10 (1971), 694-696.

[KLL] H. König, D. R. Lewis and P. K. Lin, Finite dimensional projection constants, Studia Math. 75 (1983), 341-358.

[KT1] H. König and N. Tomczak-Jaegermann, Bounds for projection constants and 1-summing norms, Trans. Amer. Math. Soc. 320 (1990), 799-823.

[KT2] -, 一, Norms of minimal projections, J. Funct. Anal. 119 (1994), 253-280.

[TJ] N. Tomczak-Jaegermann, Banach-Mazur Distances and Finite-Dimensional Operator Ideals, Longman Sci. Tech. and Wiley, Harlow and New York, 1989.

Mathematisches Seminar

Universität Kiel

24098 Kiel, Germany

E-mail: hkoenig@math.uni-kiel.de
Department of Mathematical and Statistical Sciences University of Alberta

Edmonton TG2 2G1, Canada E-mail: ntomczak@ellpspace.math.ualberta.ca

Received December 9, 2002

Revised version May 15, 2003 